\title{
Características clínicas dos pés de idosos portadores de diabetes mellitus tipo II
}

\author{
Clinical features of feet of elderly \\ patients with diabetes mellitus type II
}

FisiSenectus. Unochapecó Ano 1, n. 2 - Jul./Dez. 2013 p. 3-13

\section{Andriza Maria Ferenz}

Bacharel do Curso de Graduação em Fisioterapia da Universidade Comunitária da Região de Chapecó (Unochapecó).

\section{Daniela de Lurdes Leite Inácio Stuani}

Bacharel do Curso de Graduação em Fisioterapia da Universidade Comunitária da Região de Chapecó (Unochapecó).

\section{Vinícius Brandalise}

Docente do Curso de Graduação em Fisioterapia da Universidade Comunitária da Região de Chapecó (Unochapecó).

\section{Resumo}

Introdução: o diabetes mellitus é um problema de saúde pública que vem se destacando no Brasil, tanto pelo aumento da incidência quanto pelos fatores de risco encontrados para o desenvolvimento de suas complicações, dentre as quais estão os relacionados com os cuidados com os pés e hábitos de vida. Objetivo: avaliar as características clínicas dos pés de idosos diabéticos tipo II, cadastrados no CERES de Chapecó (SC). Materiais e métodos: estudo quantitativo descritivo, cuja amostra é de 193 indivíduos de ambos os sexos, idade entre 60 a 91 anos. Realizou-se investigação acerca do perfil dos entrevistados e seus conhecimentos sobre cuidados com os pés. Avaliamos as características clínicas dos pés e sensibilidade com estesiometria. Resultados: quanto ao perfil: $90 \%$ dos entrevistados possuíam baixa escolaridade, 88\% eram aposentados e 79\% possuíam renda de até um salário. 0 estado civil predominante foi de casados (51\%). Já em relação aos hábitos: 53\% usam tabaco, 72,5\% declaram-se sedentários e 88\% apresentaram doenças associadas. 0 exame diário dos pés é realizado diariamente por 86,5\%, dos indivíduos avaliados, 11\% usam calçados inadequados e somente 18,6\% cortam as unhas incorretamente. Somente 1 indivíduo apresentou ulceração. A sensibilidade mostrou-se alterada em 99,5\% dos casos. Conclusão: percebeu-se que o conhecimento acerca dos cuidados com os pés é sabido por grande parte dos indivíduos. A manipulação correta dos pés demonstrou ser a grande responsável pelo baixo índice de ulceração encontrada no estudo. Porém, foram observados altos índices de alterações clínicas.

\section{Palavras-chave}

Diabetes mellitus. Saúde do idoso. Avaliação em saúde. 


\begin{abstract}
Introduction: diabetes mellitus is a public health problem that has been increasing. Among the risk factors found for the development of its complications are those related to foot (care) and lifestyle. Objective: to evaluate the clinical characteristic of the feet of elderly type II diabetic patients registered at CERES Chapecó (SC). Materials and methods: descriptive quantitative study, in which sample is of 193 individuals from both sex aging 60 to 91 year old. It was made investigations on the interviewed profiles and their knowledge about feet care. We assessed the clinical characteristics of the feet and sensetivety with the esthesiometry. Results: the interviewed $90 \%$ had low scholarity, (88\%) were retired and $(79 \%)$ lived with a minimum wage. The predominante marital status was of $(51 \%)$ married, already used tabacco $(53 \%)$, sedentary $(72,5 \%)$, and the ones tha showed associated illness (88\%). The ones that examine the feet $(86,5 \%)$, wear inadequated shoes $(11 \%)$, cut the nails improperly $(18,6 \%)$, show ulcer $(0,5 \%)$. Neuropathics characteristics $(66 \%)$, ischemics (34\%), normal sensitivity alterated in (99,5\%). Conclusion: we noted that in our research that the knowledge about the feet care is known for most part of the individuals. The correct feet handling showed to be the chiefly responsable for the smallest index of ulcer cases in the study. But high rates were observed for clinical changes.
\end{abstract}

\title{
Keywords
}

Diabetes mellitus. Health of the elderly. Health evaluation.

\section{Introdução}

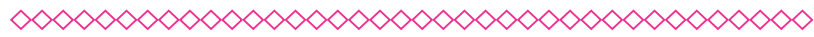

O diabetes Mellitus (DM) tem se destacado como um significativo problema de Saúde Pública, quando observado o envelhecimento demográfico da população e os fatores de risco relacionados com o processo de desenvolvimento ${ }^{1}$.

Com o envelhecimento ocorrem modificações nas estruturas anatômicas e fisiológicas dos pés, que podem vir a dificultar a deambulação e interferir na qualidade de vida da pessoa idosa, surgindo problemas decorrentes de doenças sistêmicas, alterações da marcha, maus-tratos com os pés ou mesmo traumatismos, além de condições locais podológicas, como as calosidades ${ }^{2}$.

Dentre os fatores de risco encontrados para o desenvolvimento de complicações causadas pelo diabetes estão os relacionados aos membros inferiores, e a complicação mais comum é o pé diabético, que se caracteriza por lesões cutâneas e de planos profundos, geralmente relacionados a alterações neuropáticas ${ }^{3}$. Essas alterações afetam o sistema nervoso periférico devido a desmielinização segmentar dos nervos, levando a um retardo na velocidade de condução dos estímulos sensitivos, predispondo o pé a traumas e infecções que poderão desencadear o pé diabético, evoluindo na maior parte dos casos em amputação dos membros inferiores ${ }^{4}$.

Considerando o aumento percentual de idosos portadores de diabetes mellitus tipo II, o comprometimento dos pés e suas temíveis consequências, o Ministério da Previdência e Assistência Social, por intermédio da Política Nacional do Idoso, sugere programas preventivos realizados por uma equipe multidisciplinar com pacientes diabéticos, visando identificar o pé em risco, ou seja, os que apresentem problemas neurológicos ${ }^{4}$. É importante salientar que considerável parcela de diabéticos com insensibilidade desconhecem as próprias deficiências (40\%), sendo fundamental orientá-los quanto aos cuidados que esses devem ter com os pés ${ }^{5}$. Essa prevenção é de extrema utilidade, pois, além de favorecer o autocuidado, prevenindo incapacidades, diminuir a morbidade e a mortalidade, reduz os altos custos que as complicações normalmente demandam ${ }^{6,7}$.

Os objetivos deste estudo foram avaliar as características clínicas dos pés de idosos portadores de diabetes mellitus tipo II cadastrados no Centro de Referência em Saúde (CERES) de Chapecó (SC), caracterizar o perfil desses indivíduos e avaliar o conhecimento desses em relação aos cuidados com os pés. 


\section{Materiais e métodos}

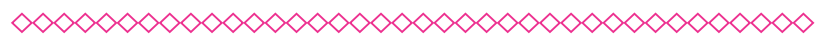

A população foi composta por 998 idosos diabéticos cadastrados no Centro de Referência em Saúde (CERES) de Chapecó (SC). A amostra se constituiu de 193 idosos de ambos os sexos; número obtido a fim de ter erro amostral de 5\%, com intervalo de confiança de $95 \%$. A média de idade foi de 71,5 anos (DP: $\pm 7,74$ ); a idade mínima foi de 60 anos, enquanto que a máxima foi de 91 anos.

A pesquisa realizada tratou-se de um estudo descritivo transversal com abordagem quantitativa dos achados.

Iniciou-se esta por meio da buscas ativas por idosos portadores de diabetes mellitus tipo II cadastrados no CERES, que caracterizou a população; após obtenção do número da amostra, foram sorteados os idosos participantes, que através de contato telefônico aceitaram participar da pesquisa. Os referidos pacientes ou seus representantes legais foram esclarecidos acerca da importância do estudo no âmbito da saúde pública. 0 formulário foi exposto e esclarecido, assim como a participação foi de livre arbítrio. 0 documento de consentimento prévio foi exibido e a autorização dos pacientes ficou expressa neste documento.

Foram incluídos no estudo portadores de diabetes mellitus tipo II, com idade acima de 60 anos. Foram excluídos da amostra indivíduos que não concordassem em participar da avaliação, que apresentassem neoplasias em membros inferiores, doença neurológica que comprometia a função nervosa periférica e cognitiva, e sua ausência no dia da visita domiciliar. Uma demonstração prévia dos procedimentos aplicados foi realizada antes da avaliação.

Utilizou-se para coleta de dados formulário para caracterização do perfil dos portadores de diabetes mellitus tipo II, adaptado de Laurindo ${ }^{8} \mathrm{e}$ Cosson, Oliveira e Adan ${ }^{9}$, ficha de avaliação dos pés de portadores de diabetes mellitus tipo II, adaptada de Bandeira ${ }^{10}$, Douat ${ }^{11}$ e Vedolin ${ }^{12}$. Os formulários foram compostos por perguntas fechadas, próprios para investigação e armazenamento das informações.
Inicialmente, realizou-se investigação acerca do perfil dos entrevistados e o conhecimento destes sobre os cuidados com os pés; na sequência, foram avaliadas as características clínicas destes pés.

Para avaliar a sensibilidade superficial protetora plantar, foi utilizado o estesiômetro, um conjunto de seis monofilamentos de náilon de Semmes-Weinstein $(0,05 \mathrm{~g}, 0,2 \mathrm{~g}, 2,0 \mathrm{~g}, 4,0 \mathrm{~g}, 10,0 \mathrm{~g}$ e $300,0 \mathrm{~g})$. 0 teste foi realizado nos dois pés, com o indivíduo sentado confortavelmente. Foram aplicados os monofilamentos em nove territórios específicos do nervo tibial posterior, começando com o monofilamento de menor peso $0,05 \mathrm{~g}$, seguindo ordem crescente até o monofilamento em que 0 indivíduo sentisse todos os pontos aplicados. 0 filamento foi aplicado três vezes em cada local do teste, sendo que uma única resposta positiva foi suficiente para confirmar sensibilidade no nível indicado. A percepção de cada monofilamento é associada a um nível funcional. Sensibilidade normal do pé (monofilamentos de 0,05g e 0,02g), diminuição da sensibilidade protetora do pé (2,0g), perda de sensibilidade protetora do pé vulnerável a lesão (monofilamento de 4,0g), perda de sensibilidade protetora do pé, podendo sentir pressão profunda (monofilamento $10,0 \mathrm{~g}$ ), sensação de pressão profunda presente (monofilamento de 300,0g), perda da sensação de pressão profunda (não sentiu com nenhum) $)^{11}$.

A avaliação do grau de comprometimento dos pés em relação ao desenvolvimento de úlceras foi realizada por meio da classificação de Wagner, que é graduada de 0 a 5 . 0 grau 0 é o pé em risco, presença de fissura interdigital ou no calcâneo, sem infecção aparente. Grau 1 apresenta infecção superficial micótica e/ou bacteriana leves. 0 grau 2 corresponde a uma infecção profunda, atingindo o tecido celular subcutâneo, tendões e ligamentos, sem osteomielite. No grau 3 ocorre infecção profunda, com abcessos na região média do pé, com tendinite ou sinovite purulentas e osteomielite. O grau 4 apresenta infecção e gangrena localizada em dedos, região plantar, anterior e calcanhar. 0 grau 5 é acometido por infecção e gangrena de todo pé, sendo o mais grave ${ }^{14}$.

0 presente estudo teve aprovação do Comitê de Ética em Pesquisa da Universidade Comunitá- 
ria da Região de Chapecó sob parecer número n. 265/09. Os resultados são apresentados em números e percentual, em forma de tabelas e gráficos.

\section{Resultados}

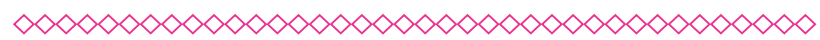

No presente estudo foram avaliados 193 idosos, $70,5 \%$ do sexo feminino e $29,5 \%$ do sexo masculino. A faixa etária variou entre 60 (mínima) a 91 (máxima) anos, com uma média de 72,5 anos (DP: $\pm 7,73)$. A maior incidência de diabetes foi encontrada nos idosos com idade entre 60 a 69 anos $(47,15 \%)$. Em relação à etnia, prevaleceram indivíduos caucasianos (76\%). Quanto ao estado civil, $51 \%$ eram casados, $9 \%$ separados, $40 \%$ viúvos e $0,5 \%$ solteiros; entre os separados e viúvos, $13,5 \%$ moravam sozinhos.

Verificou-se um predomínio de idosos com baixa escolaridade, sendo $23 \%$ analfabetos, $67 \%$ com ensino fundamental incompleto, $4,5 \%$ com ensino fundamental completo, $4,5 \%$ com ensino médio e $1 \%$ com ensino superior.

Dos entrevistados, $88 \%$ da amostra foi constituída por beneficiados do INSS (aposentadoria ou pensão), constatação já esperada devido à faixa etária acima de 60 anos. A renda de até um salário mínimo prevaleceu em $79 \%$ dos indivíduos. Na tabela 1 apresentam-se os dados sociodemográficos da população pesquisada.

Os dados relacionados ao estilo de vida mostraram que $19 \%$ dos indivíduos eram tabagistas, $34 \%$ ex-tabagistas e $47 \%$ nunca fumaram. Esses resultados nos mostram que mais da metade dos entrevistados fizeram uso de tabaco em algum momento das suas vidas. Quanto ao uso de bebida alcoólica, 85,5\% afirmaram nunca terem feito uso.

Dos idosos entrevistados, $72,5 \%$ eram sedentários, ou seja, não praticam qualquer atividade física. Em relação às comorbidades, 88\% apresentam doenças crônicas associadas, sendo a mais comum a hipertensão arterial sistêmica (69\%), seguida de hipercolesterolemia (31,5\%) e dislipidemias (14\%). Dos praticantes de atividade física, $17 \%$ não apresentaram doença associada. Na tabela 2 , são demonstrados os resultados do estilo de vida.
Quanto aos cuidados com os pés, a grande maioria (87\%) afirmou examinar os pés diariamente. Apenas $11 \%$ faz uso de calçados e/ou meias inadequadas e $19 \%$ cortam as unhas incorretamente. Os dados acima são apresentados no gráfico 1.

A presença de úlcera no nosso estudo teve um resultado insignificante quando relacionado ao número de avaliados. Apenas $0,5 \%(n=1)$ apresentou úlcera na região do calcâneo, sendo grau 1 de acordo com a classificação de Wagner.

Em relação às características clínicas dos pés, observamos a prevalência de características neuropáticas e isquêmicas, que foram observadas de acordo com a coloração, pele, unhas, deformidades, temperatura, pulsos, sensibilidade, queixas e presença de ulceração. Quanto à coloração, 68\% apresentaram-se normal ou avermelhada (coloração neuropática) e 32\% pálido/cianótico e rubor (coloração isquêmica). A pele dos avaliados, na sua maioria (63\%), se apresentou seca, com fissuras e/ ou calosidades (característica neuropática), 27\% ausência ou redução de pelos (característica isquêmica) e $10 \%$ não apresentou alterações na pele.

$\mathrm{Na}$ avaliação das unhas, 59\% apresentaram características neuropáticas (sem alterações) e 41\% características isquêmicas (atróficas, grossas, infecção fúngica).

Deformidades nos pés se encontraram presentes em $12 \%$ dos indivíduos, sendo que esta é característica do pé neuropático (dedos em garras, queda das cabeças dos metatarsos e outras deformidades), e a grande maioria (88\%) não apresentaram alterações, característico de pé isquêmico. Em relação à temperatura, 34\% apresentaram-se isquêmicos (frio) e $66 \%$ neuropáticos (quente). 0 pulso diminuído ou ausente esteve presente em $46 \%$ dos indivíduos, sendo esse característico de pé isquêmico; $54 \%$ apresentaram pulso presente, característico de pé neuropático.

As queixas foram em grande número neuropáticas, com $88 \%$ (32\% diminuída e 56\% parestesia/ queimação), e $22 \%$ isquêmicas ( $9 \%$ presente, $3 \%$ dor tipo claudicação).

Na avaliação da presença de sensibilidade superficial protetora plantar, realizada com os monfilamentos de Semmes Weinstein, os valores 
encontrados na estesiometria foram de $0,5 \%$ dos indivíduos ( 1 indivíduo com idade igual a 70 anos) com sensibilidade normal do pé (monofilamento 0,2g), 10\% (20 indivíduos, 10 com idade entre 6069 anos, 8 entre $70-79$ anos e 2 com 80 anos acima) apresentaram sensibilidade protetora diminuída (monofilamento 2,0g), 80\% apresentaram perda de sensibilidade protetora, sendo que, desses 80\%, 52\% (99 indivíduos, 50 com idade entre 60-69 anos, 37 entre 70-79 anos e 12 com 80 anos acima) apresentaram sensibilidade com o monofilamento de 4,0g e $28 \%$ (54 indivíduos, 24 com idade entre 60-69 anos, 20 com 70-79 anos, 10 com 80 anos acima) sentiram com o monofilamento de $10,0 \mathrm{~g}$. Oito por cento (16 indivíduos, 5 com idade entre 60-69 anos, 4 com 70-79, 7 com 80 anos acima) tiveram sensação de pressão profunda presente (monofilamento 300,0g) e 1,5\% (1 com idade entre 60-69 anos, 2 com 80 anos acima) perda da sensação de pressão profunda (anestesia plantar). Esses resultados demonstraram que a sensibilidade normal do pé está alterada em 99,5\% dos indivíduos, a perda de sensibilidade protetora está na grande maioria (39\%) em indivíduos com idade entre 60 a 69 anos, seguida dos indivíduos com idade entre 70 a 79 anos (30\%). A sensação de pressão profunda foi encontrada com índice maior $(4,5 \%)$ nos indivíduos com idade igual ou superior a 80 anos. No gráfico 2 estão dispostos os dados relacionados a estesiometria.

Partindo da observação das características clínicas dos pés e da aplicação dos monofilamentos, podemos constatar que $66 \%$ dos indivíduos apresentaram características de pé neuropático, enquanto $34 \%$ apresentaram características de pé isquêmico.

\section{Discussão}

$\infty \times \infty \times \infty \times \infty \times \infty \times \infty \times \infty \times \infty \times \infty \times \infty \times \infty \times \infty \times \infty \times$

0 envelhecimento não começa subitamente aos 60 anos, mas consiste no acúmulo e interação de processos bio-psico-socioculturais durante toda a vida que podem constituir fatores de adoecimento ou promotores de saúde e de bem-estar ${ }^{15}$.

Estudos realizados ${ }^{16}$ reforçam os dados encontrados em nossa pesquisa em relação a etnia, sen- do a maior prevalência de diabetes em indivíduos caucasianos.

Nossa amostra foi constituída por grande parte de indivíduos com baixa escolaridade ( $90 \%)$. Estudos brasileiros realizados com população diabética adulta encontraram maiores frequências entre os níveis de escolaridade do ensino fundamental incompleto e de analfabetismo, apontando ser esta variável dificultadora no processo de cuidado a uma pessoa com doença crônica ${ }^{18}$. A baixa escolaridade dificulta o processo de ensino e aprendizagem, e pessoas que não tiveram acesso à educação possuem maiores riscos de desenvolverem complicações em membros inferiores ${ }^{18}$.

Em relação ao tabagismo, os dados encontrados em nosso estudo são confirmados pela literatura, que identificam que as pessoas fumantes têm maiores chances de sofrerem consequências negativas nos membros inferiores, como amputação, tendo 4,6 vezes mais chances em relação aos não fumantes ${ }^{19}$. Quanto ao hábito de ingerir bebida alcoólica, não foi verificada correlação com a ocorrência de complicações para membros inferiores.

Quanto às doenças crônicas associadas, foram encontrados estudos ${ }^{20}$ que corroboram com nossa pesquisa, sendo as doenças que mais se destacam a hipertensão arterial e o colesterol.

Foi observado o efeito do exercício físico regular no controle glicêmico em pacientes com diabetes mellitus tipo II, no controle do colesterol total e da hipertensão arterial sistêmica ${ }^{21}$, sendo a atividade física mencionada como instrumento de recuperação, manutenção e promoção saúde ${ }^{22}$.

Estudo refere que a maioria das pessoas diabéticas considera importante o cuidado com os pés na prevenção de complicações, mas apenas 50\% os examinam diariamente ${ }^{23}$. Observamos, porém, que os indivíduos avaliados, na sua maioria (87\%), têm o hábito de examinar os pés diariamente, assim como conhecem os cuidados que se deve ter com os pés, o que nos faz perceber que o baixo índice de ulceração encontrado se deu pelo fato desses terem consciência da importância dos cuidados com os pés, prevenindo assim complicações como as infecções e as úlceras. Os dados encontrados na nossa pesquisa divergem de outros estu- 
dos realizados ${ }^{18}$ em relação à baixa escolaridade, como já exposto acima. Nosso estudo apresentou um grande índice de indivíduos com baixa escolaridade $(90 \%)$, demonstrando que a falta de estudo não teve influência no processo de aprendizagem e no cuidados com os pés.

As justificativas encontradas em nosso estudo para as dificuldades em relação ao exame dos pés se relacionaram com o avanço da idade, o que dificulta a inspeção dos pés e ausência do apoio familiar.

As úlceras isquêmicas ocorrem preferencialmente em regiões marginais do pé, submetidas à pressão contínua (por exemplo: calçados apertados), podendo evoluir para isquemia e gangrena digital; nos pés neuropáticos aparecem com mais frequência em pontos de alta pressão na região plantar, em geral onde existem calosidades ${ }^{24}$.

Observamos em nosso estudo um índice elevado de indivíduos com ressecamento nos pés (63\%). Em estudo realizado ${ }^{25} \mathrm{com} 225$ pacientes diabéticos, percebeu-se que o comprometimento autonômico leva a um ressecamento dos pés e, em alguns pacientes, desencadeia fissuras plantares, sendo considerada a diminuição ou ausência de sudorese nos pés fator contribuinte para a constituição de úlceras plantares.

No processo de envelhecimento ocorrem alterações funcionais da pele, tais como ressecamento, frouxidão, afilamento e fragilidade, perda de elasticidade etc. Trabalhos que investigam a função neurossensorial da pele do idoso concluem que a percepção de uma série de estímulos diminui com a idade ${ }^{26}$.

As micoses e infeç̧ões do pé são condições agravantes dos transtornos neuropáticos, isquêmicos ou de ambos, levando ao descontrole metabólico ou progressão para infecção generalizada ${ }^{4}$. Os pacientes diabéticos geralmente apresentam visão comprometida pela retinopatia diabética, e ao tentarem cortar sozinhos os calos e os cantos das unhas acabam ferindo a pele, que passa a ser porta de entrada para uma infecção $0^{5}$.

A parestesia, quase sempre encontrada no exame físico, traduz um grau de comprometimento neurológico, com alterações importantes da sensibilidade que, muitas vezes, levam a aneste- sia local ${ }^{27}$. 0 paciente diabético com neuropatia periférica queixa-se de parestesia, hiperestesia e dor, muitas vezes lancinante, já no pé isquêmico a dor a princípio ocorre ao caminhar (claudicação), podendo chegar à dor em repouso, que piora com a elevação dos membros ${ }^{25}$. Os dados acima corroboram com nosso estudo, no qual se observou que a parestesia está presente em grande parte (56\%) da população diabética pesquisada.

Em estudo feito com 27 diabéticos, por intermédio dos monofilamentos, observou-se que a sensibilidade normal dos pés estava presente em $11 \%$ (3 indivíduos), diminuição da sensibilidade protetora em $25,9 \%$, perda da sensibilidade protetora em $7,4 \%$, presença de sensibilidade à pressão profunda em $22,2 \%$ e $33,3 \%$ não responderam a nenhum monofilamento ${ }^{28}$.

0 pé, como estrutura anátomo-funcional, é de fundamental importância no aparelho locomotor, e está relacionado não somente com a capacidade de suporte do peso corporal, mas também com a adequada distribuição dessa carga, além de funcionar como proprioceptor periférico, já que orienta o controle funcional do indivíduo e faz a recepção de sensações térmicas, táteis e de pressão ${ }^{29}$.

A perda da sensibilidade sensorial, a diminuição do número de receptores neurossensoriais periféricos e a diminuição da velocidade de condução nervosa no nervo periférico estão associadas com o processo de envelhecimento e estabelecem correlações clínicas e funcionais a esses achados ${ }^{30}$.

Os dados encontrados em nosso estudo e em outros trabalhos pesquisados nos mostraram que a sensibilidade plantar nos diabéticos está associada também com a idade, descontrole metabólico e a longa evolução da doença que levam os portadores de diabetes a apresentarem neuropatia periférica, com diminuição da sensibilidade dos pés, da formação de calos e, consequentemente, da formação de úlceras ${ }^{7}$.

A avaliação da sensibilidade com uso do monofilamento possui um papel fundamental para detecção precoce dos distúrbios da função motora. A incapacidade em identificar estímulos dolorosos ou qualquer outra lesão da extremidade inferior resulta na perda de um importante mecanismo protetor; a perda deste sinal faz com que problemas relativamente simples possam progredir rapi- 
damente para situações que chegam a ameaçar o membro ${ }^{11}$.

\section{Conclusão}

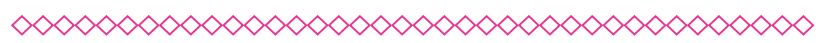

Após a aplicação dos formulários e testes específicos, observamos que o perfil dos diabéticos avaliados foi de indivíduos com baixo nível socioeconômico e cultural. Embora 99,5\% dos indivíduos tenham apresentado alterações consideráveis de sensibilidade, percebemos que o conhecimento acerca dos cuidados com os pés é sabido por grande parte dos sujeitos avaliados e que esse conhecimento, assim como a conscientização dos pesquisados em fazer uso dos seus conhecimentos em relação aos cuidados e higiene dos pés, é responsável pelo baixo índice de ulceração encontrada no estudo.

Salientamos a importância de ações e campanhas educativas a essa população diabética, no sentido de aumentar a preservação das extremidades inferiores, diminuindo, com isso, o elevado custo para a sociedade relacionado às complicações do diabetes, além de prevenir futuras incapacidades e auxiliando na melhora da qualidade de vida dessa população.

\section{Referências}

$\infty_{\infty}+\infty \times \infty \times \infty \times \infty \times \infty \times \infty \times \infty \times \infty \times \infty \times \infty \infty$

1. Brasil. Ministério da Saúde. Cadernos de Atenção Básica - n. 16: Diabetes mellitus. Brasília - DF, 2006.

2. Rocco JCP. Avaliação do Pé Geriátrico e sua Relação com Quedas. [Dissertação] Faculdade de Medicina, Universidade de São Paulo, São Paulo, 2000.

3. Gamba MA. Amputações por diabetes mellitus: uma prática prevenível. Acta Paul Enferm, São Paulo, v. 11, n. 3, p. 92-100, ago, 1998.

4. Calsolari MR, Castro RF, Maia RM, Maia FCP, Castro AV, Reis R, et al. Análise Retrospectiva dos Pés de Pacientes Diabéticos do Ambulatório de Diabetes da Santa Casa de Belo Horizonte, MG.
Arq Bras Endocrinol Metab, São Paulo; v.46, n.2, 2002.

5. Maffei FHA, Lastória S, Yoshida WB, Rollo HÁ. Doenças vasculares periféricas. v.2, 3.ed., Rio de Janeiro: Medsi; 2002.

6. Milman M. Pé Diabético: avaliação da evolução e custo hospitalar de pacientes internados no conjunto hospitalar de Sorocaba, 2001. Arq Bras Endocrinol Metab, São Paulo, v. 45, n. 5, p. 447451, out. 2001.

7. Lopes CF. Projeto de assistência ao pé do paciente portador de diabetes mellitus. J Vasc Bras, v.2, n. 1, Nov, 2003.

8. Laurindo MC. Conhecimento das pessoas diabéticas acerca dos cuidados com os pés. Arq Ciênc Saúde, abr-jun, v.12, n.2, 2005.

9. Cosson ICO, Oliveira FN, Adan LF. Avaliação do conhecimento de medidas preventivas do pé diabético em pacientes de Rio Branco, Acre. Arq Bras Endocrinol Metab, Botucatu, v. 49, n. 4, 2005.

10. Bandeira F. Endocrinologia e diabetes. Rio de janeiro: Medsi; 2003.

11. Douat S. Avaliação do uso de monofilamentos para prevenção do pé diabético. Rev Bras Fisioter, Rio de Janeiro, v. 3, n. 3, p.157-163, mai/jun, 2002.

12. Vedolin AC. Pé Diabético: estudo comparativo entre diferentes formas de apresentação clínica e tratamentos. Serviço de angiologia e cirurgia vascular do Hospital de Clínicas da Universidade Federal do Paraná, 2003.

13. Rocha MJ, Cunha EP, Dinis A, et al. Feridas uma Arte Secular - Avanços Tecnológicos no Tratamento de Feridas. 2 ed. Goiânia : Coimbra; 2006.

14. Sizínio H. Ortopedia e traumatologia: princípios e prática. 3. ed. Porto Alegre: Artmed, 2003.

15. Garcia MAA, Odoni APC, Souza CS, Frigerio RM, Merlin SS. Idosos em cena: falas do adoecer. Interface Comunic Saúde Educ, Botucatu. v.9, n. 18, p. 537-589, 2005. 
16. Ramos LR, Rosa TE, Oliveira Z, Medina MCG, Santos FRG. Perfil do idoso em área metropolitana na região sudeste do Brasil: resultado de inquérito domiciliar. Rev Saúde Pública, v.27, n.2, 1993.

17. Modeneze DM. Qualidade de vida e diabetes: limitações físicas e culturais de um grupo específico. [Dissertação] Campinas: Faculdade de Educação Física, Universidade Estadual de Campinas; 2004.

18. Pace $A E$, Foss MC. Fatores de risco para complicações em extremidades inferiores de pessoas com diabetes mellitus. Rev Bras Enferm, Brasília, v. 55, n. 5, Set-Out. 2002.

19. Gamba MA, Gotlieb SL, Bergamaschi DP, Vianna, LAC. Amputações de Extremidades Inferiores por Diabetes Mellitus: Estudo CasoControle. Rev Saúde Pública, v.38, n.3, 2004.

20. Raskin DBF. Menopausa, obesidade, gordura corporal e fatores de risco para doenças cardiovasculares. [Dissertação]. Campinas: Faculdade de Educação Física, Universidade Estadual de Campinas; 2000.

21. Fernandes CAM, Carolino IDR, Elias RGM, Nardo, JN, Tasca RS, Cuman, RKN. Efeito do exercício aeróbico sobre o perfil lipídico de pacientes idosas, portadoras de Diabetes Mellitus tipo 2, atendidas em Unidade Básica de Saúde, Maringá, Estado do Paraná. Rev Bras Geriatr Gerontol, v.11, n.2, Rio de Janeiro; 2008.

22. Matsudo SM, Matsudo VR, Araujo T, Andrade $D$, Andrade E, Oliveira L, Braggion G. Nível de atividade física da população de São Paulo: análise de acordo com o gênero, idade nível sócio econômico, distribuição geográfica e de conhecimento. Rev Bras Ciênc Mov, Brasília v.10, n.4, out, 2002.

23. Barbui EC, Cocco MIM. Conhecimento do cliente diabético em relação aos cuidados com os pés. Rev Esc Enferm, USP. São Paulo; v.36, n.1, 2002.

24. Rodrigues CDS. A contribuição do diabetes mellitus nas amputações de membros inferiores. [Dissertação]. Ribeirão Preto: Universidade de São Paulo; 2003.

25. Barreira AA. Classificação das neuropatias diabéticas. Rev Bras Neurol, v.27, n.1; 1991.

26. Souza A, Nery CAS, Marciano LHSC, Garbino JA. Avaliação da neuropatia periférica: Correlação entre a sensibilidade cutânea dos pés, achados clínicos e eletromiográficos. Acta Fisiátrica, São Paulo, v.12, n.3, p.87-93, 2005.

27. Foss NT, Foss MC. Lesões plantares do paciente diabético - pé diabético. Rev Bras Neurol. v.27, n.1, 1991

28. Fregonesi CET, Faria CRS, Molinari SL. Avaliação Sensitiva do Nervo Tibial através de Monofilamentos em Portadores de Diabetes Mellitus. Arq Ciênc Saúde, v.6, n.2, mai-ago, 2002.

29. Fabrício SCC, Rodrigues RAP, Junior MLC. Causas e consequências de quedas de idosos atendidos em hospital público. Rev Saúde Pública, São Paulo, v.38, n.1, p.93-102, 2004.

30. Delisa JA, Gans BM. Rehabilitation Medicine: Principles and Practice. Philadelphia, v.1, p.660667, 1993. 


\section{Anexos}

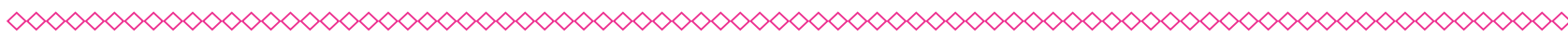

Tabela 1 - Perfil do portadores de diabetes mellitus: dados sociodemográficos

\begin{tabular}{|c|c|c|c|}
\hline & & Percentual (\%) & Número $(\mathrm{N})$ \\
\hline \multirow[t]{2}{*}{ Sexo } & Feminino & 70,5 & 136 \\
\hline & Masculino & 29,5 & 57 \\
\hline \multirow[t]{3}{*}{ Idade } & $60-69$ anos & 47,2 & 91 \\
\hline & 70-79 anos & 36,2 & 70 \\
\hline & 80 ou mais & 16,6 & 32 \\
\hline \multirow[t]{4}{*}{ Estado civil } & Solteiro & 0,5 & 1 \\
\hline & Casado & 51 & 98 \\
\hline & Separado & 8,5 & 17 \\
\hline & Viúvo & 40 & 77 \\
\hline \multirow[t]{2}{*}{ Etnia } & Branco & 76 & 147 \\
\hline & Negro & 24 & 46 \\
\hline \multirow[t]{5}{*}{ Escolaridade } & Analfabeto & 23 & 44 \\
\hline & $\begin{array}{l}\text { Ensino fundamental } \\
\text { incompleto }\end{array}$ & 67 & 129 \\
\hline & $\begin{array}{l}\text { Ensino fundamental } \\
\text { completo }\end{array}$ & 9 & 18 \\
\hline & Ensino médio & 0,5 & 1 \\
\hline & Curso superior & 0,5 & 1 \\
\hline \multirow[t]{2}{*}{ Benefícios (aposentadoria, pensão) } & Sim & 88 & 170 \\
\hline & Não & 22 & 23 \\
\hline \multirow[t]{4}{*}{ Renda mensal } & Sem renda & 9 & 18 \\
\hline & Até 1 salário & 79 & 151 \\
\hline & 1 a 3 salários & 9 & 18 \\
\hline & Mais de 3 salários & 3 & 6 \\
\hline
\end{tabular}

Fonte: elaboração dos autores. 
Tabela 2 - Perfil dos portadores de diabetes mellitus tipo II: estilo de vida

\begin{tabular}{llcc} 
& & Percentual (\%) & Número (N) \\
Tabagista & Sim & 19 & 37 \\
& Não & 34 & 66 \\
& Ex-tabagista & 47 & 90 \\
\hline Etilista & Sim & 2,5 & 5 \\
& Não & 85,5 & 165 \\
& Ex-etilista & 12 & 23 \\
\hline Pratica exercícios físicos & Sim & 27,5 & 53 \\
& Não & 72,5 & 140 \\
\hline Doenças associadas & Sim & 88 & 151 \\
& Não & 22 & 43 \\
\hline
\end{tabular}

Fonte: elaboração dos autores.

(clique para voltar ao texto)

Tabela 3 - Características clínicas do pés dos portadores de diabetes mellitus

\begin{tabular}{llrlc} 
Coloração & \multicolumn{1}{c}{ Isquêmico } & \multicolumn{2}{c}{ Neuropático } \\
& Rubor & $18,00 \%$ & Normal/avermelhado & $68,00 \%$ \\
Pele & Ausência/redução de pelos & $27,00 \%$ & $\begin{array}{l}\text { Seca/fissuras e/ou } \\
\text { calosidades }\end{array}$ & $63,00 \%$ \\
& Atróficas/grossas/infeç̧ões & $41,50 \%$ & Sem alterações & $58,5 \%$ \\
\hline Unhas & fúngicas & & & \\
\hline Deformidade & Ausente & $88,00 \%$ & Presente & $12,00 \%$ \\
\hline Temperatura & Frio & $34,00 \%$ & Quente & $66,00 \%$ \\
\hline Pulsos & Diminuído/ausente & $46,00 \%$ & Presente & $54,00 \%$ \\
\hline Sensibilidade & Presente & $0,50 \%$ & Diminuída/ausente & $99,50 \%$ \\
\hline Queixas & Presente & $9,50 \%$ & Diminuída/ausente & $32,00 \%$ \\
& Dor tipo & $2,50 \%$ & Parestesia/anestesia/ & $56,00 \%$ \\
& Claudicação/repouso & & queimação/lancinante & \\
\hline Úlceras & Em geral nas regiões & $0,50 \%$ & Em geral plantar & $0,00 \%$ \\
& marginais e dedos & & & \\
\hline TOTAL & Isquêmico & $34,00 \%$ & Neuropático & $66,00 \%$ \\
\hline
\end{tabular}

Fonte: elaboração das autoras. 
Gráfico 1 - Conhecimento dos indivíduos pesquisados em relação aos cuidados com os pés.

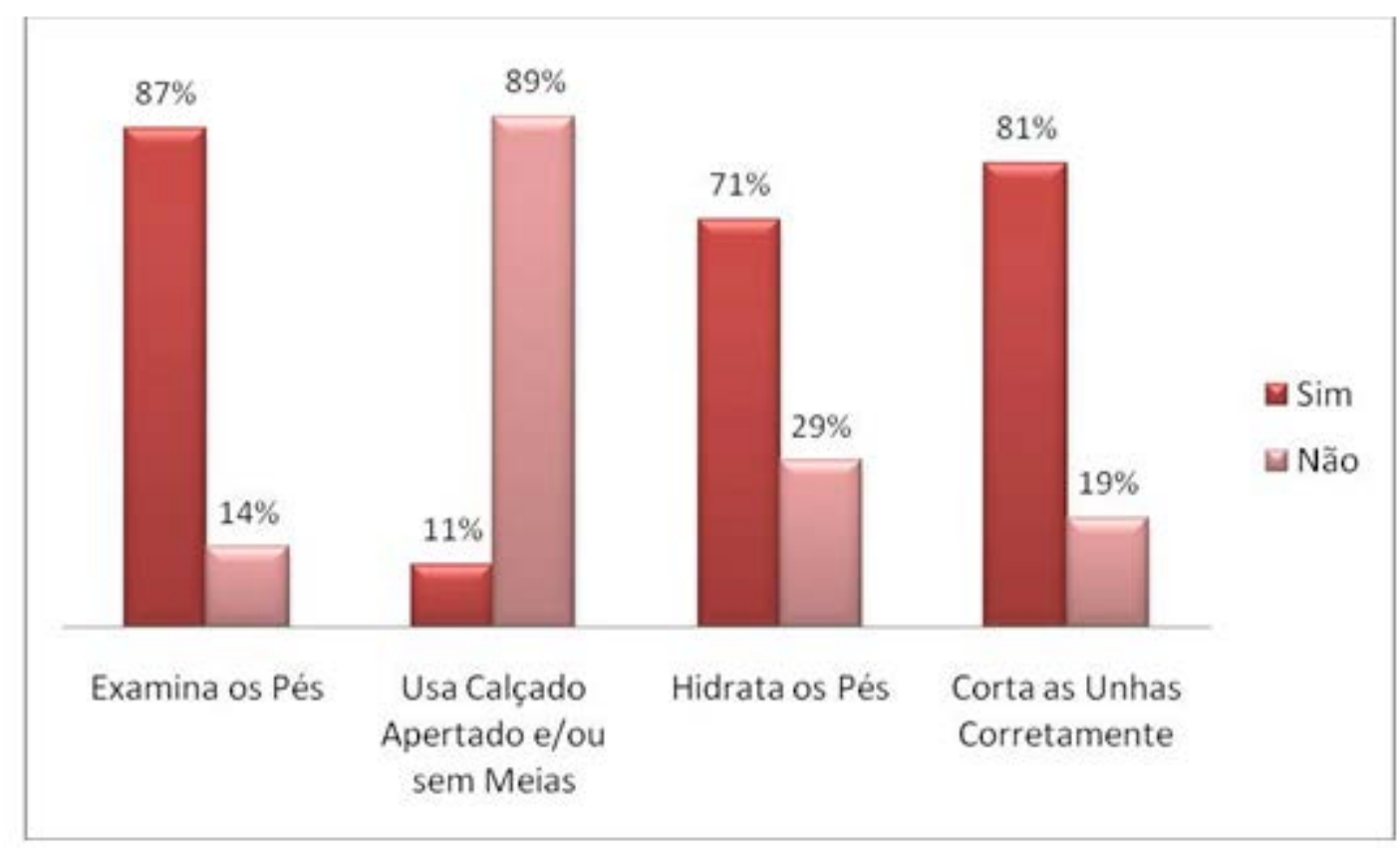

Fonte: elaboração dos autores.

(clique para voltar ao texto)

Gráfico 2 - Presença de sensibilidade superficial protetora plantar, separada por monifilamentos e feixa etária.

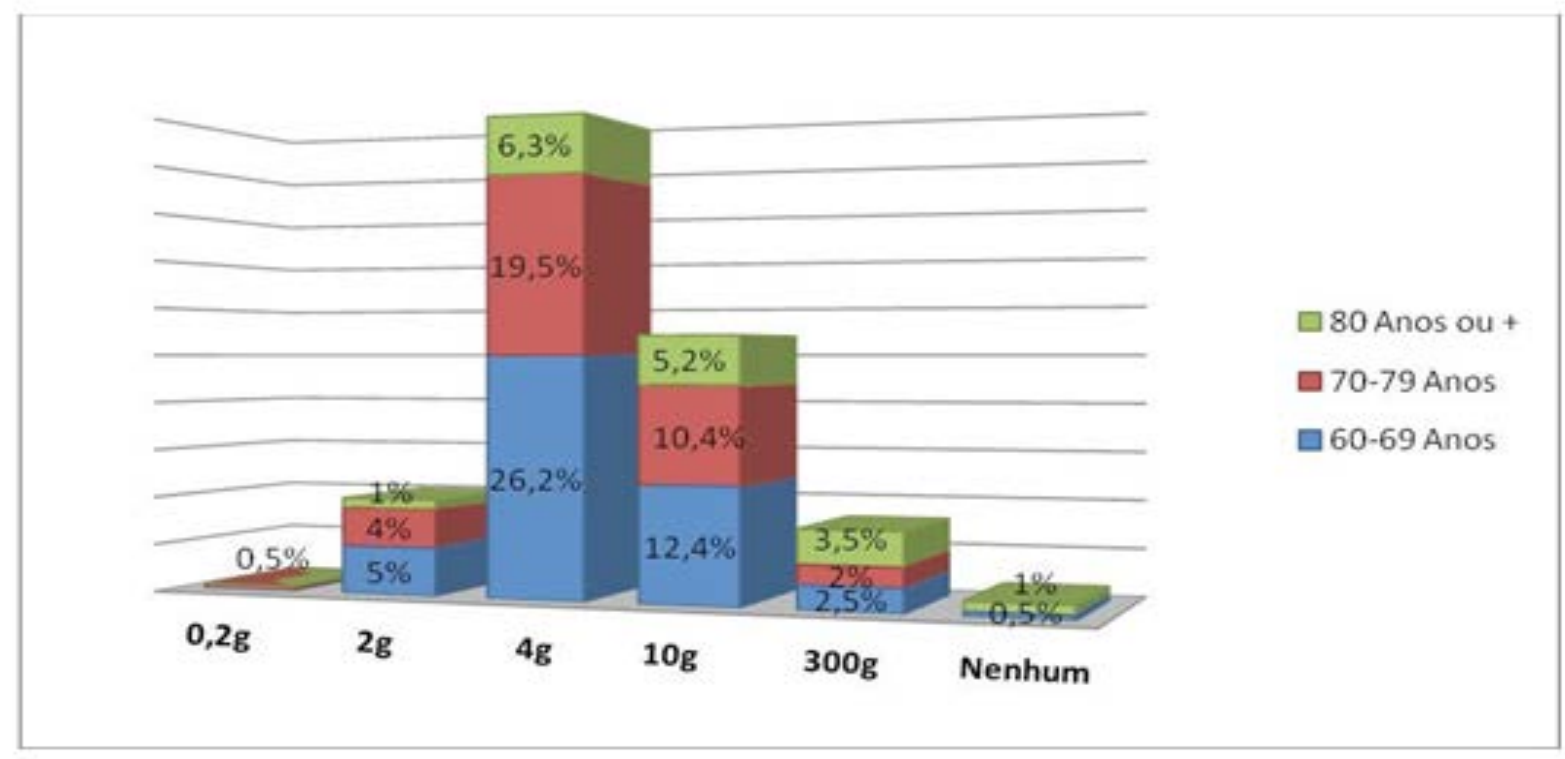

Fonte: elaboração dos autores.

(clique para voltar ao texto) 